\title{
Algoritmo Evolucionário com Inspiração Quântica e Sequências Caóticas para Seleção de Atributos em Interfaces Cérebro-Computador
}

\author{
Alimed Celecia ${ }^{1}$, Marley Vellasco ${ }^{1}$ \\ ${ }^{1}$ Departamento de Engenharia Elétrica - Pontifícia Universidade Católica de Rio de \\ Janeiro (PUC-Rio) \\ Caixa Postal 22451-900 - Rio de Janeiro - RJ - Brazil \\ alimedcr22@gmail.com, marley@ele.puc-rio.br
}

\begin{abstract}
In this paper we explore the effects of adding ergodicity to a Quantum-inspired Evolutionary Algorithm (QiEA) by the employment of chaotic maps in two operators: chaotic uniform crossover and chaotic quantum update gate. The proposed algorithm is validated in the feature selection stage of a Brain-Computer Interfaces (BCI) application using Electroencephalography (EEG) of imagined right and left hand movement. The results are compared with the ones of a QiEA and a classical Genetic Algorithm (GA). The results demonstrated that Chaotic QiEAs can significantly improve the convergence time of the model with only a small loss in the final accuracy.
\end{abstract}

Resumo. Este trabalho descreve a exploração dos efeitos de adicionar ergodicidade a um Algoritmo Evolucionário com inspiração Quântica (QiEA) utilizando sequências caóticas em dois operadores: crossover uniforme caótico e porta quântica de atualização caótica. O algoritmo é aplicado na seleção de atributos de uma aplicação de interface cérebro-computador (BCI) que emprega o eletroencefalograma (EEG) da imaginação do movimento das mãos direita ou esquerda. Os resultados são comparados com os de um QiEA e Algoritmo Genético (GA) tradicionais, demonstrando que o QiEA caótico pode aperfeiçoar significativamente o tempo de convergência do modelo com só uma pequena perda na acurácia final do modelo.

\section{Introdução}

A translação de teorias procedentes das ciências da natureza ao âmbito da Inteligência Computacional tem gerado algoritmos capazes de resolver inúmeros problemas. Um exemplo da convergência de duas áreas do conhecimento como a Física e a Biologia no domínio da computação são os Algoritmos Evolucionários com inspiração Quântica (QiEA) (Zhang, 2011). Esse tipo de algoritmos tem demonstrado uma efetividade sobressalente na solução de problemas em diversas aplicações abrangendo saúde (González, Vellasco and Figueiredo, 2019), seleção de atributos (Ramos and Vellasco, 2018) e Neural Architecture Search (Szwarcman, Civitarese and Vellasco, 2019).

Os QiEA integram conceitos da computação quântica como qubits, portas quânticas, e coerência para aumentar a eficiência do processo de busca. Como consequência, entre suas principais vantagens se encontram o balanceamento entre exploitation e 
exploration, a capacidade de, com uma população reduzida, encontrar o máximo/mínimo global (Kuk-Hyun Han and Jong-Hwan Kim, 2002), e uma grande velocidade de convergência (Wang et al., 2013). Não obstante, o algoritmo apresenta uma alta sensitividade à variação da direção e magnitude do ângulo de rotação (Ramos and Vellasco, 2018), sendo afetada diretamente a capacidade e tempo de convergência deste.

Uma técnica para melhorar a taxa de convergência e a velocidade dos algoritmos evolucionários consiste na adição de componentes caóticos em diversas partes do algoritmo. Algoritmos como Differential Evolution (Senkerik et al., 2018), ou Algoritmos Genéticos (GA) (Fuertes et al., 2019) tem se beneficiado da ergodicidade das sequencias caóticas. No entanto, QiEAs tem sido raramente explorados com essa técnica apesar das características caóticas dos qubits (Hui, Jiashu and Chao, 2004). Especificamente, (Hui, Jiashu and Chao, 2004), (Zhao et al., 2009), e (Mozaffari et al., 2017), propuseram novos operadores como a porta quântica, mutação, e crossover caóticos para incrementar capacidade de convergência e a performance do algoritmo em comparação a algoritmos genéticos tradicionais.

Uma das aplicações em que os QiEA apresentam uma vantagem significativa é a seleção de atributos em interfaces cérebro-computador (BCI). Este tipo de dispositivos realizam a translação de sinais cerebrais em comandos de controle para algum equipamento externo. No caso mais comum de abordagem não invasiva, a sinal de eletroencefalografia (EEG) adquirida durante a realização ou imaginação de certa tarefa é processada com o objetivo de detectar a intenção do usuário (Lotte et al., 2018). O processo envolve principalmente técnicas de Inteligência Computacional para realizar o pré-processamento do sinal, o cálculo de atributos, a seleção de atributos, e finalmente a classificação.

Os sistemas de BCI requerem uma performance elevada e uma rápida convergência devido a que, para cada usuário, o sistema deve ser retreinado. Essa característica, em combinação com a não linearidade, sensibilidade a artefatos, e variações no tempo do sinal de EEG para diferentes usuários ou incluso para o mesmo usuário em diferentes sessões, estabelece a necessidade de um pipeline de processamento que seja à vez o mais rápido e acurado possível.

Num trabalho prévio nós propusemos uma abordagem wrapper baseada num QiEA com representação binaria para a seleção de atributos em aplicações de BCI (Ramos and Vellasco, 2018). Os resultados obtidos pelo algoritmo para três usuários realizando uma tarefa de imaginação motora (IM) do movimento das mãos direita ou esquerda foram superiores aos de um GA tradicional. Durante os experimentos, foi reportado a sensitividade do algoritmo à magnitude do ângulo de rotação, resultando em drásticas variações na velocidade de convergência e a performance general.

Sendo assim, neste trabalho temos como objetivo descobrir os efeitos de adicionar ergodicidade ao QiEA com representação binária mediante a utilização de sequencias caóticas (Nagashima, Baba and Nakahara, 1999) para definir o operador de recombinação e a porta quântica de atualização dos qubits. Adicionalmente, o algoritmo será aplicado na etapa de seleção de atributos numa abordagem wrapper para a classificação de sinais EEG de IM, o qual, até o alcance de nosso conhecimento, não tem sido abordado na literatura. O algoritmo QiEA caótico (CQiEA) proposto será avaliado comparando a acurácia de uma rede neural de múltiplas camadas (MLP) 
treinada utilizando sinais de três usuários disponíveis nos dados públicos das Competições de BCI II e III com a de um GA tradicional e o QiEA de (Ramos and Vellasco, 2018).

Este trabalho se encontra ordenado como descrito a continuação: a próxima seção descreve cada metodologia utilizada no pipeline de processamento do sinal de EEG, focando nos modelos propostos neste trabalho (sistemas caóticos e QiEAs). Seção 3 introduze os dados dos usuários utilizados para avaliar o CQiEA e os resultados obtidos pelos diferentes modelos, discutindo ademais sua relevância. Finalmente, na Seção 4 são apesentados os comentários finais sobre o trabalho.

\section{Metodologias}

O modelo BCI proposto segue a mesma estrutura descrita em (Celecia et al., 2017; Ramos and Vellasco, 2018). As quatro etapas (resumidas na Figura 1) e as metodologias envolvidas em cada uma delas constituem o objeto de estudo das seguintes subseções.

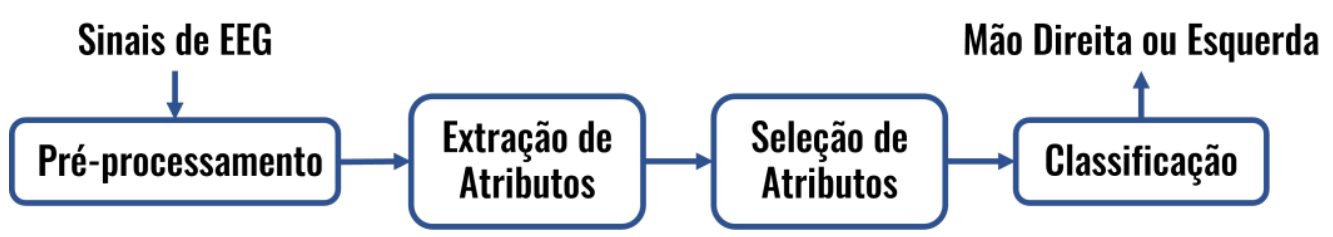

Figura 1. Etapas do sistema de $\mathrm{BCl}$ utilizado

\subsection{Pré-processamento e Extração de Atributos}

Sinais de EEG são caracterizadas por sua aleatoriedade, variações extremas no tempo, e não-estacionaridade. Como consequência, o pré-processamento desse tipo de sinais requere o tratamento do sinal para eliminar componentes indesejadas ou ruidosas e artefatos musculares ou eletrocutares, e melhorar o Signal to Noise Ratio (SNR) (Graimann, Allison and Pfurtscheller, 2009). Também, durante essa etapa os sinais são representados de forma mais adequada para a extração de características mediante a aplicação de técnicas de análises temporais ou espectrais (ou alguma combinação delas).

Um método de análise tempo-frequência recomendado para sinais aleatórias e nãoestacionarias é a Wavelet Packet Decomposition (WPD) (Ting et al., 2008). A aplicação desta técnica permite não só a representação do sinal simultaneamente nos domínios temporais e espectrais, mas também separa as componentes do sinal nas diferentes subbandas de frequência de interesse. Em (Celecia, González and Vellasco, 2016) pode se ler uma explicação mais detalhada dessa técnica.

No modelo de BCI proposto, a WPD é aplicada utilizando a mother wavelet Daubechie de ordem $4(\mathrm{db} 4)$ e quatro níveis de decomposição. O sinal resultante no último nível se encontra decomposto em 16 sub-bandas de uma largura de banda de $4 \mathrm{~Hz}$. Dentre essas sub-bandas, para representar os sinais de EEG, são selecionadas oito bandas na faixa 0$32 \mathrm{~Hz}$.

Para representar o sinal de cada sub-banda, sobre os sinais reconstruídos são calculadas uma série de informações estatísticas, de potência e de fase delas. Mais detalhes sobre as definições dos atributos são descritos em (Celecia et al., 2017). O conjunto de atributos final possui 134 atributos (inclui os canais de EEG C3 e C4). 


\subsection{Seleção de atributos}

Devido à alta dimensionalidade do vetor de atributos e visando selecionar um conjunto de atributos com uma alta acurácia na etapa de classificação, e alta velocidade no fluxo de processamento foi introduzida uma etapa de seleção de atributos no sistema de BCI. Algoritmos para seleção de atributos podem ser classificados em filters e wrappers (Liu et al., 2010) dependendo da forma de avaliação dos atributos ou conjunto de atributos. A categoria de filters abrange aqueles algoritmos que utilizam um critério baseado nas características dos dados, como information gain ou redundância, e que seja totalmente independente do modelo de aprendizado. Os wrappers, por outro lado, realizam a avaliação de subconjuntos de atributos a partir de métricas obtidas do modelo de aprendizado como acurácia ou precisão.

Em nosso trabalho anterior (Ramos and Vellasco, 2018) foi proposto um wrapper baseado num QiEA com representação binaria que superou significativamente os resultados de um GA tradicional na taxa de convergência para a classificação de sinais de EEG de IM. Apesar das vantagens desse tipo de modelo, a magnitude do ângulo de rotação para a porta quântica de atualização possui um impacto relevante no tempo de convergência do algoritmo e na performance final. Uma alternativa de solução para esta limitação é a adição de comportamento caótico no QiEA mediante a utilização de sequencias caóticas, gerando versões caóticas do operador de crossover e da porta quântica de atualização dos indivíduos. O modelo de CQiEA binário proposto, até onde sabemos, nunca tem sido aplicado para seleção de atributos, aplicações de BCI, ou processamento de sinais de EEG.

\subsubsection{QiEA Tradicional}

QiEAs com representação binária estão baseados nos qubits como substitutos dos bits clássicos. Estes, num sistema quântico, existem numa superposição coerente dos estados binários tradicionais. Por conseguinte, podem representar não só os estados $|0\rangle$ e $|1\rangle$, mas também a superposição deles (Kuk-Hyun Han and Jong-Hwan Kim, 2002). O estado de um qubit é descrito como:

$$
|\psi\rangle=\alpha|0\rangle+\beta|1\rangle
$$

onde $\alpha$ e $\beta$ são valores complexos denominados amplitude de probabilidade do sistema se encontrar num dos estados clássicos. Devido à propriedade unitária das magnitudes probabilísticas estes valores devem cumprir com a condição $|\alpha|^{2}+|\beta|^{2}=1$, onde $|\alpha|^{2} \mathrm{e}$ $|\beta|^{2}$ são as probabilidades de observar os estados $|0\rangle$ ou $|1\rangle$, respectivamente.

Um cromossoma quântico é formado por um conjunto de qubits consecutivos. Este, mediante o processo de observação, colapsa da superposição de estados a um dos estados clássicos, conformando um indivíduo binário tradicional. A representação do cromossoma de $m$ qubits seria:

$$
q_{j}^{t}=\left[\begin{array}{c}
\alpha_{j 1}^{t} \\
\beta_{j 1}^{t}
\end{array}\left|\begin{array}{c}
\alpha_{j 2}^{t} \\
\beta_{j 2}^{t}
\end{array}\right| \ldots \mid \begin{array}{c}
\alpha_{j m}^{t} \\
\beta_{j m}^{t}
\end{array}\right]
$$

A população quântica $\mathrm{Q}(\mathrm{t})=\left\{q_{1}^{t}, q_{2}^{t}, \ldots, q_{n}^{t}\right\}$ é inicializada definindo para cada qubit uma amplitude de probabilidade de $1 / 2^{1 / 2}$ para os dos estados. Desta forma, é garantido igual probabilidade de observação dos estados no início do processo de busca. Como 
operador de crossover é utilizado o crossover uniforme. O operador principal no QiEA é a porta de rotação quântica, a qual modifica as amplitudes de probabilidades dos qubits direcionando a evolução aos estados das melhores soluções dentro da população. Esse processo é conhecido como atualização:

$$
\left[\begin{array}{c}
\alpha_{j i}^{t+1} \\
\beta_{j i}^{t+1}
\end{array}\right]=\left[\begin{array}{cc}
\cos \Delta \theta & -\sin \Delta \theta \\
\sin \Delta \theta & \cos \Delta \theta
\end{array}\right]\left[\begin{array}{c}
\alpha_{j i}^{t} \\
\beta_{j i}^{t}
\end{array}\right]
$$

onde $\Delta \theta$ é o ângulo de rotação que define a aproximação angular do estado do qubit na direção dos estados $|0\rangle$ ou $|1\rangle$. Dada a sua função, a seleção da magnitude deste ângulo é de grande importância para o sucesso final do processo de otimização. No QiEA tradicional, a evolução finaliza quando é atingido o número máximo de gerações.

\subsubsection{Sistemas caóticos}

Os sistemas caóticos são aqueles fenômenos com comportamento similar a uma série de elementos irregulares definidos por uma regra relativamente simples (Nagashima, Baba and Nakahara, 1999). Esses sistemas determinísticos e não-lineares podem ser representados como mapas ou sequencias caóticas (funções singulares ou multidimensionais nas quais o valor num ponto específico dependo só do valor no ponto anterior).

Outras formas de representar sistemas caóticos é através de atratores, os quais são padrões formados pelo sistema ao ser representado no espaço de fase. Esses sistemas dinâmicos apresentam uma alta sensibilidade às condições iniciais, pelo que variações nos parâmetros iniciais da sequência podem gerar grandes diferenças nos valores obtidos nas iterações posteriores. Também são caracterizados pelo seu comportamento pseudoaleatório e ergodicidade.

\subsubsection{CQiEA}

Dada a natureza caótica dos qubits, rápida taxa de convergência e capacidade de evitar cair nos mínimos locais ( $\mathrm{Lu}$ et al., 2014), neste trabalho é proposto substituir a aleatoriedade do QiEA por sequências caóticas. Nesta forma, desordem ergódico é acrescentado ao algoritmo para aperfeiçoar a performance do processo evolutivo (Mozaffari et al., 2017). Os novos operadores propostos são o operador de crossover caótico e a porta quântica de rotação caótica.

A modificação da probabilidade do Crossover Uniforme selecionar o gene de um dos pais pode diminuir a capacidade disruptiva do operador. $\mathrm{Na}$ abordagem proposta, definimos um valor dinâmico de probabilidade $P$ ditado pela sequência caótica $X$.

$$
P(t)=X(t-1)
$$

O segundo operador é a porta quântica de rotação caótica. O ângulo de rotação desta no QiEA tradicional pode ser mantido constante ou selecionado de uma tabela de pesquisa (a qual implica um aumento do custo computacional do algoritmo). Para o CQIEA, o valor do ângulo é proporcional ao valor da sequência caótica nessa geração:

$$
\Delta \theta(t)=\pi \cdot X(t-1)
$$


Tendo em conta a sensibilidade dos sistemas caóticos às condições iniciais, no CQiEA, após gerar as sequências caóticas, um número aleatório representa o ponto de entrada na série para geração inicial. O resultado é a geração de condições iniciais diferentes para os diferentes operadores e experimentos. No caso das sequências caóticas bidimensionais o método de dobrar o atrator sobre o eixo y (Senkerik et al., 2018) é aplicado para obter um valor único em cada geração.

A Figura 2 representa o pseudocódigo do CQiEA. Para aplicar o algoritmo no sistema de BCI proposto, os cromossomas quânticos são definidos com 134 qubits (um representando cada atributo), e cada observação gera um indivíduo clássico que representa o conjunto de atributos selecionado em função de seu valor binário. A aptidão dos indivíduos clássicos é definida pela acurácia de uma MLP com dez neurônios na camada escondida selecionada de cinco modelos treinados com inicialização aleatória dos pesos. Um diagrama simplificado das etapas principais do CQiEA aplicado na seleção de atributos é ilustrado na Figura 3.

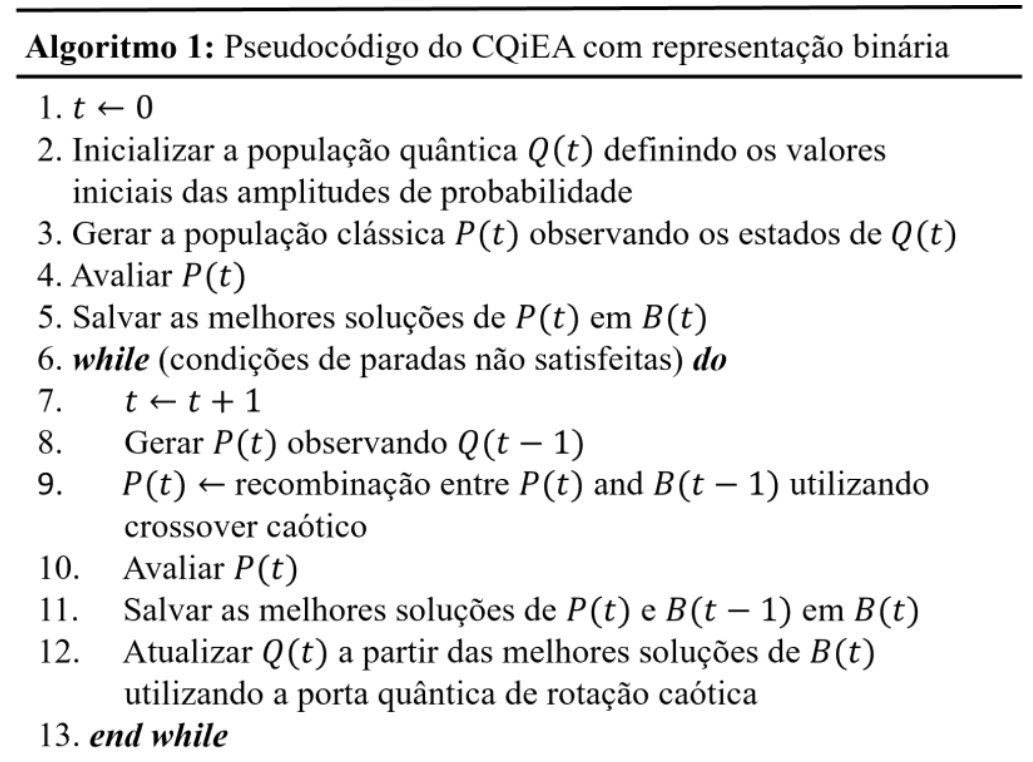

Figura 2. Pseudocódigo do CQiEA com representação binária

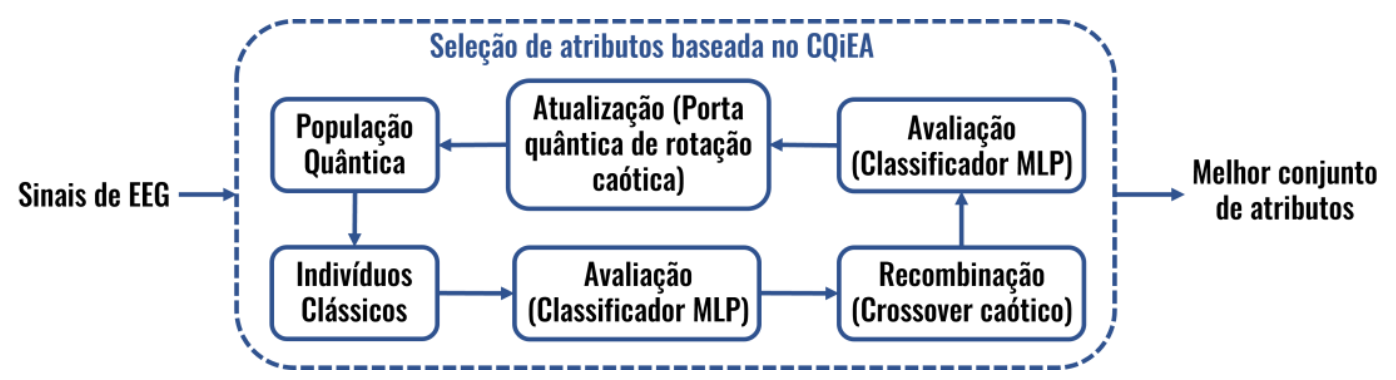

Figura 3. Diagrama simplificado da aplicação do CQiEA para a seleção de atributos

As sequências caóticas escolhidas para este trabalho foram seis, incluindo unidimensionais e bidimensionais. Delas, a partir da avaliação dos resultados obtidos, pode ser escolhida a que representa o melhor mapa para nossa aplicação. Uma breve descrição dos mapas pode ser observada na Tabela 1 . 
Tabela 1. Definições das sequências caóticas utilizadas neste trabalho

\begin{tabular}{|c|c|c|}
\hline $\begin{array}{c}\text { Sequências } \\
\text { caóticas }\end{array}$ & Definição & Parâmetros \\
\hline \multirow{2}{*}{ Burgers map } & $X_{n+1}=a X_{n}-Y_{n}{ }^{2}$ & $a=0.75$ \\
& $Y_{n+1}=b Y_{n}+X_{n} Y_{n}$ & $b=1.75$ \\
\hline \multirow{2}{*}{ Henon map } & $X_{n+1}=a-X_{n}{ }^{2}+b Y_{n}$ & $a=1.4$ \\
& $Y_{n+1}=X_{n}$ & $b=0.3$ \\
\hline \multirow{3}{*}{ Ikeda map } & $X_{n+1}=\gamma+\mu\left(X_{n} \cos \varphi+Y_{n} \sin \varphi\right)$ & $\alpha=6$ \\
& $Y_{n+1}=\mu\left(X_{n} \cos \varphi+Y_{n} \sin \varphi\right)$ & $\beta=0.4$ \\
& $\varphi=\beta-\alpha /\left(1+X_{n}{ }^{2}+Y_{n}{ }^{2}\right)$ & $\gamma=1$ \\
\hline Logistic map & $X_{n+1}=a X_{n}\left(1-X_{n}\right)$ & $\mu=0.9$ \\
\hline Quadratic map & $X_{n+1}=a-X_{n}{ }^{2}$ & $a=4$ \\
\hline & $X_{n+1}=X_{n}{ }^{2}-Y_{n}{ }^{2}+a X_{n}+b Y_{n}$ & $a=1.95$ \\
\hline \multirow{3}{*}{ Tinkerbell map } & $Y_{n+1}=2 X_{n} Y_{n}+c X_{n}+d Y_{n}$ & $a=0.9$ \\
& & $c=0.6$ \\
& & $d=0.5$ \\
\hline
\end{tabular}

\subsection{Classificação}

Para a etapa de classificação foi implementada uma MLP com topologia dinâmica. Especificamente, o modelo de rede neural implementa uma variação do número de neurônios na camada escondida entre 5 e 20, sendo cada rede treinada 5 vezes para escolher a que obteve melhor acurácia. No âmbito das BCI, esse modelo classificador é considerado altamente acurado e robusto (Lotte et al., 2018). A diferença deste modelo em comparação ao utilizado na seleção de atributos é a topologia variável, a qual permite uma exploração melhor do espaço de configurações possível para gerar uma acurácia final superior.

\section{Resultados}

Os dados utilizados neste trabalho foram fornecidos pelas BCI Competitions II (Dataset III-MI) e III (Dataset IIIb-MI) (Schlögl, 2002, 2005). O conjunto de dados estão formados pelos sinais de EEG de três usuários que realizaram tarefas de MI incluindo as mãos direita e esquerda. Os sinais foram filtrados entre 0.5 e $30 \mathrm{~Hz}$. A Tabela 2 fornece mais detalhes sobre estes dados.

Todos os algoritmos foram desenvolvidos e testados usando o software Matlab 2016. Para facilitar o processo de classificação, os dados foram normalizados entre 0 e 1 e os dados de treino foram divididos em $70 \%$ das instancias para treino e 30\% para validação. O CQiEA foi configurado com 5 indivíduos quânticos, 8 observações para cada indivíduo (para um total de 40 indivíduos clássicos), e 40 gerações. Os valores são mantidos como em (Ramos and Vellasco, 2018) para permitir estabelecer uma comparação direta com os resultados prévios. 
Tabela 2. Características dos dados

\begin{tabular}{|c|c|c|c|c|c|}
\hline Dataset & Usuários & $\begin{array}{c}\text { Duração do } \\
\text { experimento }\end{array}$ & \multirow{2}{*}{ Eletrodos } & $\begin{array}{c}\text { Frequência de } \\
\text { amostragem }\end{array}$ & $\begin{array}{c}\text { Instancias de } \\
\text { Treino/Test }\end{array}$ \\
\hline III-MI & $\mathrm{A} 2$ & $9 \mathrm{~s}$ & \multirow{2}{*}{$\mathrm{C} 3, \mathrm{C} 4$} & $128 \mathrm{~Hz}$ & $140 / 140$ \\
\cline { 1 - 2 } IIIb-MI & $\mathrm{S} 4$ & \multirow{2}{*}{$7 \mathrm{~s}$} & & $125 \mathrm{~Hz}$ & $540 / 540$ \\
\cline { 2 - 2 } & $\mathrm{X} 11$ & & & $540 / 540$ \\
\hline
\end{tabular}

Duas vertentes foram estabelecidas para analisar os resultados. A primeira visa discutir as características do processo evolucionário para cada usuário. A segunda tem como objetivo destacar os resultados para os melhores modelos no conjunto de teste.

As Figuras 4, 5 e 6 mostram os resultados, para os três sujeitos, da performance obtida pelas sequências caóticas avaliadas no CQiEA, e a do QiEA apresentada em (Ramos and Vellasco, 2018) e um GA tradicional sobre o conjunto de validação. As curvas da evolução ilustram a notável taca de convergência do CQiEA e sua capacidade de alcançar o máximo global. Para os sujeitos S4 e X11, os mapas Ikeda e Logistic causaram uma convergência mais acelerada em comparação com o QiEA e GA tradicionais, atingindo também maiores valores de performance. O número de avaliações requeridas para obter a melhor performance foi reduzido num 55\% (de 968 para 440 avaliações) para o mapa Logistic no usuário S4 e cerca de 39\% (de 520 para 320 avaliações) para o mapa Ikeda no usuário X11. A performance máxima foi aperfeiçoada em cerca de $1 \%$ para S4 e $1.5 \%$ para X11.

Para o usuário A2 resultados similares aos anteriores foram obtidos para os mapas Logistic e Tinkerbell. Efetivamente, a abordagem proposta não só aumenta a performance, mas também decrementa o número de avaliações necessário para conseguir a performance máxima.

Dos mapas caóticos testados, o que forneceu os melhores resultados foi o Logistic, seguido pelo Ikeda. Em geral, para os três sujeitos, o Logistic resultou numa melhora considerável, sendo o segundo melhor tanto em tempo de convergência como em performance para os usuários X11 e A2, e o melhor para S4. O mapa Ikeda apresentou resultados sobressalientes para X11 e foi o segundo melhor para S4, mas para A2 seus resultados foram muito pobres. A sinergia do mapa Logistic e o QiEA está em concordância com as conclusões reportadas em (Mozaffari et al., 2017).

As outras sequências caóticas tiveram um comportamento marcadamente variado para os diferentes usuários. Para S4 e X11, só o mapa Quadratic apresentou uma performance superior ao QiEA, mas com uma convergência mais demorada. Um caso particular é o do mapa Tinkerbell, o qual foi o melhor para A2 e nos outros usuários a performance foi baixa.

A acurácia média e o desvio padrão dos resultados obtidos no conjunto de teste para o conjunto de atributos selecionado pelo algoritmo com 10 rodadas do CQiEA utilizando os mapas Logistic e Ikeda, e o QiEA e GA tradicionais são mostrados na Tabela 3. Em geral, a acurácia para as abordagens CQiEA são relativamente menores que os valores obtidos para o QiEA e o GA. Isto pode estar relacionado à própria natureza do modelo wrapper, o qual pode sofrir de overfitting ao otimizar para um conjunto de validação, limitando a capacidade de generalização do conjunto de atributos selecionado. No entanto, a velocidade de convergência do CQiEA é muito superior, pelo que, para a aplicação em questão, tem de ser considerado se se deseja priorizar a velocidade do 
treinamento ou a seleção de um modelo computacionalmente mais custoso para um ganho pequeno de acurácia. Também, ao ter uma grande velocidade de convergência, seria possível aplicar métodos para limitar o overfitting como alguma variante de validação cruzada sem ter um aumento considerável no tempo de treino.

As vantagens de introduzir as sequências caóticas nos QiEAs se resumem em dois aspectos. Primeiramente, ao utilizar os dois novos operadores caóticos na recombinação e atualização dos indivíduos, o balance entre exploration e exploitation é melhorado, permitindo direcionar a busca em novos espaços. Em segundo lugar, a velocidade de convergência e significativamente aperfeiçoada, permitindo ao processo de busca encontrar soluções de melhor qualidade (ex: novos valores de acurácia máxima para S4 e X11).

S4

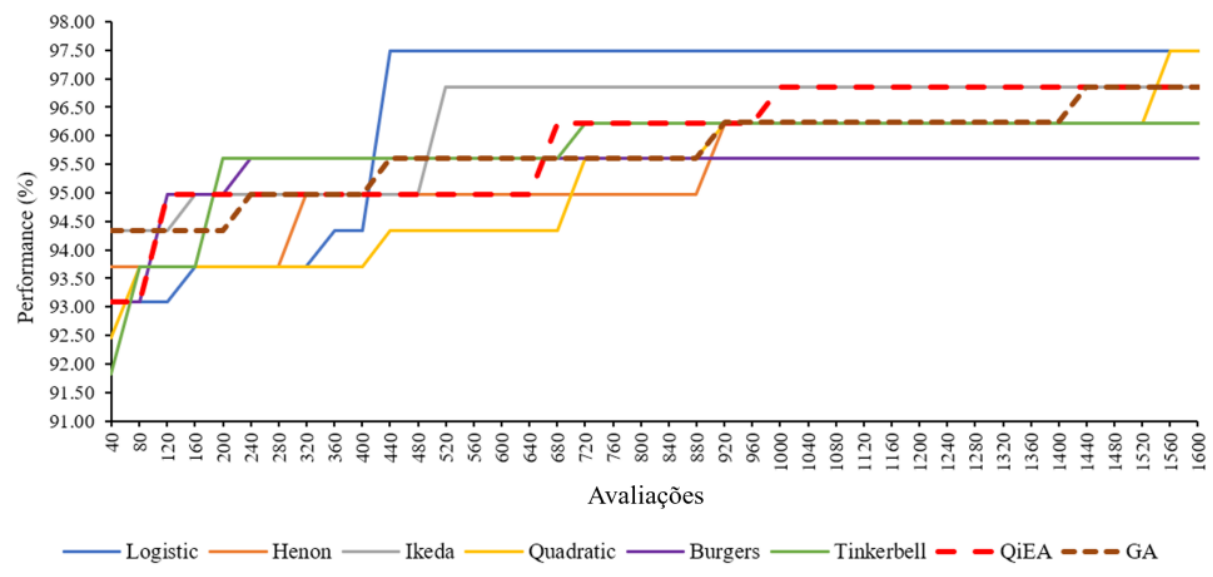

Figura 4. Resultados da seleção de atributos para o usuário S4 para os diferentes mapas caóticos e o CQiEA, o QiEA tradicional e um GA

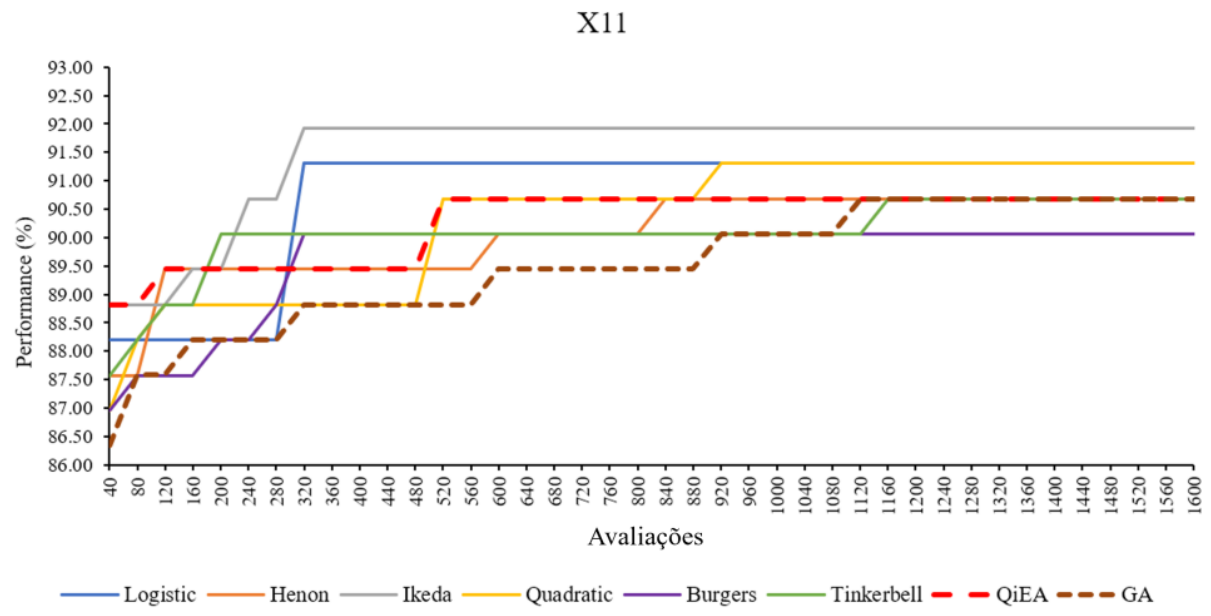

Figura 5. Resultados da seleção de atributos para o usuário X11 para os diferentes mapas caóticos e o CQiEA, o QiEA tradicional e um GA 


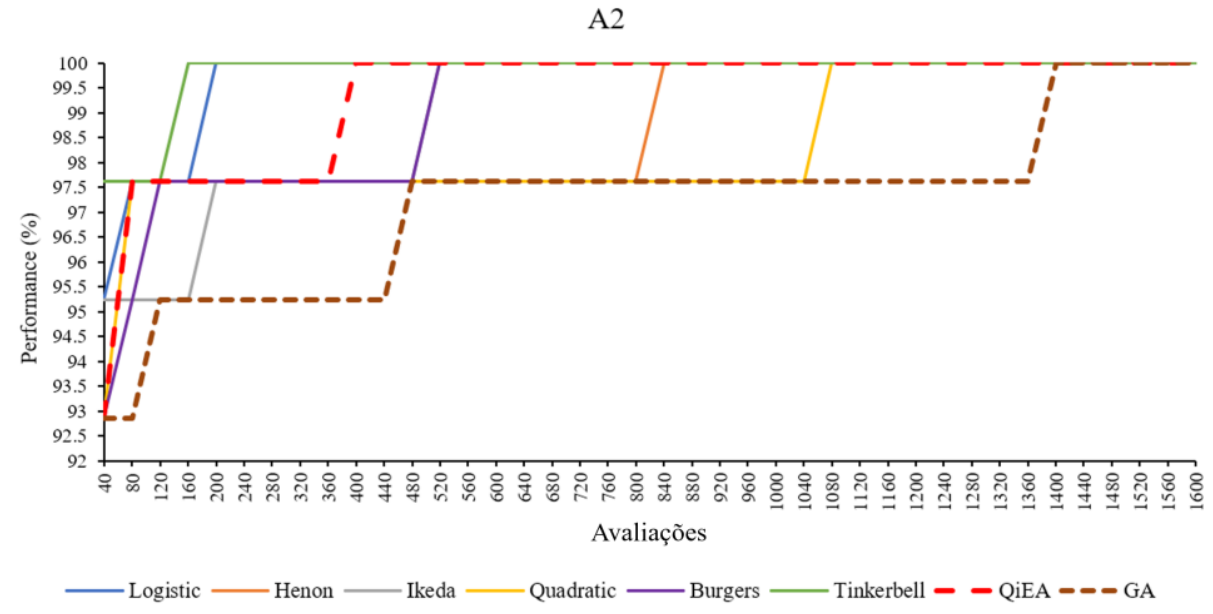

Figura 6. Resultados da seleção de atributos para o usuário A2 para os diferentes mapas caóticos e o CQiEA, o QiEA tradicional e um GA

Tabela 3. Resultados do melhor conjunto de atributos para as melhores abordagens de CQIEA, QIEA e GA

\begin{tabular}{|c|c|c|c|c|c|c|}
\hline & \multicolumn{2}{|c|}{ S4 } & \multicolumn{2}{c|}{ X11 } & \multicolumn{2}{c|}{ A2 } \\
\hline & $\begin{array}{c}\text { Acurácia } \\
\text { Média }\end{array}$ & $\begin{array}{c}\text { Desvio } \\
\text { Padrâo }\end{array}$ & $\begin{array}{c}\text { Acurácia } \\
\text { Média }\end{array}$ & $\begin{array}{c}\text { Desvio } \\
\text { Padrâo }\end{array}$ & $\begin{array}{c}\text { Acurácia } \\
\text { Média }\end{array}$ & $\begin{array}{c}\text { Desvio } \\
\text { Padrâo }\end{array}$ \\
\hline CQiEA - Logistic & 87.88 & 0.98 & 81.96 & 0.95 & 95.43 & 1.30 \\
\hline CQiEA - Ikeda & 89.27 & 1.47 & 82.52 & 0.76 & 94.71 & 0.64 \\
\hline QiEA & 89.82 & 1.05 & 83.62 & 0.89 & 95.29 & 0.92 \\
\hline GA & 89.84 & 0.98 & 83.74 & 0.74 & 95.00 & 0.78 \\
\hline
\end{tabular}

\section{Conclusões}

Neste trabalho, nós investigamos os efeitos de adicionar ergodicidade a um QiEA com representação binária mediante a utilização de sequências caóticas para criar variantes caóticas de crossover e porta quântica de rotação caótica com aplicação na seleção e atributos num sistema de BCI. O trabalho partiu da hipótese de que, dada a evidência das vantagens da combinação de sistemas caóticos e algoritmos evolucionários, o QiEA também se beneficiaria dessa abordagem. A nossa principal motivação se baseou em que, aos sistemas de BCI precisar de treinamento individual para cada uso, uma abordagem wrapper com tempo reduzido de processamento que consiga manter um nível alto de acurácia na classificação da intenção do usuário poderia ser de grande utilidade. $\mathrm{O}$ algoritmo proposto foi avaliado empregando dados de três usuários disponíveis publicamente, incluindo abordagens baseadas em 6 diferentes sequências caóticas. Os resultados obtidos foram comparados também com os resultados do QiEA e GA tradicionais. A qualidade do algoritmo em combinação com cada sequência caótica foi determinada utilizando uma MLP como modelo classificador.

Os resultados obtidos demonstraram que, dependendo do mapa caótico utilizado (sendo os melhores o Logistic ou Ikeda), se pode atingir a performance máximo com uma velocidade de convergência maior que o QiEA e o GA clássicos. Ao avaliar a qualidade do conjunto de atributos escolhido para novos dados, foi percebida um decaimento pequeno na acurácia, a qual pode ser solucionada mediante modificações do processo de 
aprendizado (por exemplo, incluindo alguma variante de validação cruzada). Os experimentos mostraram que, com um tempo de convergência muito menor, o CQiEA pode ser efetivo na seleção de atributos de um sistema de BCI.

Como trabalhos futuros, as limitações percebidas na capacidade de generalização do modelo serão estudadas e serão exploradas variantes do algoritmo para limitar o possível overfitting do modelo final. Adicionalmente, será estudada a implementação de um modelo híbrido com a adição de representação real numa aplicação neuroevolutiva que, além de selecionar os atributos, defina os parâmetros do modelo de aprendizado e pré-processamento. Finalmente, as vantagens deste algoritmo para aplicações de BCI serão estudadas num modelo online que inclua dados de um capacete para aquisição de EEG e uma órtese para reabilitação após derrame cerebral.

\section{Referências}

Celecia, A. et al. (2017) 'Ensemble of classifiers applied to motor imagery task classification for BCI applications', in 2017 International Joint Conference on Neural Networks (IJCNN). IEEE, pp. 2995-3002. doi: 10.1109/IJCNN.2017.7966227.

Celecia, A., González, R. and Vellasco, M. (2016) 'Feature Selection Methods Applied to Motor Imagery Task Classification', in LA-CCI 2016 Latin American Conference on Computational Intelligence. Cartagena, Colombia.

Fuertes, G. et al. (2019) 'Chaotic genetic algorithm and the effects of entropy in performance optimization', Chaos. American Institute of Physics Inc., 29(1). doi: $10.1063 / 1.5048299$.

González, R., Vellasco, M. and Figueiredo, K. (2019) 'Resource optimization for elective surgical procedures using quantum-inspired genetic algorithms', in Proceedings of the Genetic and Evolutionary Computation Conference on - GECCO '19. New York, New York, USA: ACM Press, pp. 777-786. doi: 10.1145/3321707.3321786.

Graimann, B., Allison, B. and Pfurtscheller, G. (2009) 'Brain-Computer Interfaces: A Gentle Introduction', in Brain-Computer Interfaces. Springer Berlin Heidelberg, pp. 127. doi: 10.1007/978-3-642-02091-9_1.

Hui, C., Jiashu, Z. and Chao, Z. (2004) 'Chaos updating rotated gates quantum-inspired genetic algorithm', in 2004 International Conference on Communications, Circuits and Systems, pp. 1108-1112. doi: 10.1109/icccas.2004.1346370.

Kuk-Hyun Han and Jong-Hwan Kim (2002) 'Quantum-inspired evolutionary algorithm for a class of combinatorial optimization', IEEE Transactions on Evolutionary Computation, 6(6), pp. 580-593. doi: 10.1109/TEVC.2002.804320.

Liu, H. et al. (2010) 'Feature Selection: An Ever Evolving Frontier in Data Mining', Journal of Machine Learning Research: Workshop and Conference Proceedings 10: The Fourth Workshop on Feature Selection in Data Mining, pp. 4-13.

Lotte, F. et al. (2018) 'A review of classification algorithms for EEG-based braincomputer interfaces: A 10 year update', Journal of Neural Engineering. Institute of Physics Publishing. doi: 10.1088/1741-2552/aab2f2.

Lu, H. et al. (2014) 'The effects of using chaotic map on improving the performance of multiobjective evolutionary algorithms', Mathematical Problems in Engineering. 
Hindawi Publishing Corporation, 2014. doi: 10.1155/2014/924652.

Mozaffari, A. et al. (2017) 'Comparisons of several variants of continuous quantuminspired evolutionary algorithms', Journal of Experimental and Theoretical Artificial Intelligence. Taylor and Francis Ltd., 29(4), pp. 869-909. doi: 10.1080/0952813X.2016.1264088.

Nagashima, H., Baba, Y. and Nakahara, M. (1999) Introduction to Chaos. 1st edn, Introduction to Chaos. 1st edn. CRC Press. doi: 10.1201/9780429187001.

Ramos, A. C. and Vellasco, M. (2018) 'Quantum-inspired Evolutionary Algorithm for Feature Selection in Motor Imagery EEG Classification', in 2018 IEEE Congress on Evolutionary Computation, CEC 2018. Institute of Electrical and Electronics Engineers Inc. doi: 10.1109/CEC.2018.8477705.

Schlögl, A. (2002) 'Data set: BCI-experiment'. BCI competition 2003. Available at: www.bbci.de/competition/ii/Graz_description.doc.

Schlögl, A. (2005) 'Dataset IIIb: Non-stationary 2-class BCI data'. BCI Competition III.

Senkerik, R. et al. (2018) 'On the Population Diversity for the Chaotic Differential Evolution', in 2018 IEEE Congress on Evolutionary Computation, CEC 2018 Proceedings. Institute of Electrical and Electronics Engineers Inc. doi: 10.1109/CEC.2018.8477741.

Szwarcman, D., Civitarese, D. and Vellasco, M. (2019) 'Quantum-Inspired Neural Architecture Search', in Proceedings of the International Joint Conference on Neural Networks. Institute of Electrical and Electronics Engineers Inc. doi: 10.1109/IJCNN.2019.8852453.

Ting, W. et al. (2008) 'EEG feature extraction based on wavelet packet decomposition for brain computer interface', Measurement, 41(6), pp. 618-625. doi: 10.1016/j.measurement.2007.07.007.

Wang, H. et al. (2013) 'The improvement of quantum genetic algorithm and its application on function optimization', Mathematical Problems in Engineering, 2013(1). doi: 10.1155/2013/730749.

Zhang, G. (2011) 'Quantum-inspired evolutionary algorithms: a survey and empirical study', Journal of Heuristics. Springer US, 17(3), pp. 303-351. doi: 10.1007/s10732010-9136-0.

Zhao, S. et al. (2009) 'Real-coded chaotic quantum-inspired genetic algorithm for training of fuzzy neural networks', Computers and Mathematics with Applications, 57(11-12), pp. 2009-2015. doi: 10.1016/j.camwa.2008.10.048. 